\title{
Comparison between MRI and pathology in the assessment of tumour regression grade in rectal cancer
}

Francesco Sclafani ${ }^{1}$, Gina Brown ${ }^{2}$, David Cunningham ${ }^{*},{ }^{1}$, Andrew Wotherspoon ${ }^{3}$, Larissa Sena Teixeira Mendes ${ }^{3}$, Svetlana Balyasnikova², Jessica Evans ${ }^{2}$, Clare Peckitt ${ }^{4}$, Ruwaida Begum ${ }^{1}$, Diana Tait ${ }^{1}$, Josep Tabernero ${ }^{5}$, Bengt Glimelius ${ }^{6}$, Susana Roselló ${ }^{7}$, Janet Thomas ${ }^{1}$, Jacqui Oates ${ }^{1}$ and lan Chau $^{1}$

${ }^{1}$ Department of Medicine, The Royal Marsden NHS Foundation Trust, London and Surrey, UK; ${ }^{2}$ Department of Radiology, The Royal Marsden NHS Foundation Trust, London and Surrey, UK; ${ }^{3}$ Department of Histopathology, The Royal Marsden NHS Foundation Trust, London and Surrey, UK; ${ }^{4}$ Clinical Research \& Development, The Royal Marsden NHS Foundation Trust, London and Surrey, UK; ${ }^{5}$ Department of Medical Oncology, Vall d'Hebron University Hospital, Universitat Autònoma de Barcelona, Barcelona, Spain; ${ }^{6}$ Department of Immunology, Genetics and Pathology, Section of Experimental and Clinical Oncology, University of Uppsala, Uppsala, Sweden and ${ }^{7}$ Department of Haematology and Medical Oncology, Biomedical Research Institute INCLIVA, University of Valencia, Valencia, Spain

Background: Limited data exist regarding the correlation between MRI tumour regression grade (mrTRG) and pathological TRG (pTRG) in rectal cancer.

Methods: mrTRG and PTRG were compared in rectal cancer patients from two phase II trials (EXPERT and EXPERT-C). The agreement between radiologist and pathologist was assessed with the weighted $\kappa$ test while the Kaplan-Meier method was used to estimate survival outcomes.

Results: One hundred ninety-one patients were included. Median time from completion of neoadjuvant treatment to preoperative MRI and surgery was 4.1 weeks (interquartile range (IQR): 3.7-4.7) and 6.6 weeks (IQR: 5.9-7.6), respectively. Fair agreement was found between mrTRG and PTRG when regression was classified according to standard five-tier systems $(\kappa=0.24)$ or modified three-tier systems $(\kappa=0.25)$. Sensitivity and specificity of mrTRG 1-2 (complete/good radiological regression) for the prediction of pathological complete response was $74.4 \%$ (95\% Cl: 58.8-86.5) and 62.8\% (95\% Cl: 54.5-70.6), respectively. Survival outcomes of patients with intermediate pathological regression (pTRG 2) were numerically better if complete/good regression was also observed on imaging (mrTRG 1-2) compared to poor regression (mrTRG 3-5) (5-year recurrence-free survival $76.9 \%$ vs $65.9 \%, P=0.18$; 5 -year overall survival $80.6 \%$ vs $68.8 \%, P=0.22$ ).

Conclusions: The agreement between mrTRG and PTRG is low and mrTRG cannot be used as a surrogate of pTRG. Further studies are warranted to assess the ability of mrTRG to identify pathological complete responders for the adoption of nonoperative management strategies and to provide complementary prognostic information to PTRG for better risk-stratification after surgery.

*Correspondence: Professor D Cunningham; E-mail: david.cunningham@rmh.nhs.uk

Received 6 April 2017; revised 10 August 2017; accepted 22 August 2017; published online 21 September 2017

(C) 2017 Cancer Research UK. All rights reserved 0007-0920/17 
On the basis of the high concordance between radiological data and pathological findings, high-resolution magnetic resonance imaging (MRI) has become the gold standard for rectal cancer staging and assessment of baseline risk factors such as threatening/ involvement of the mesorectal fascia and extramural venous invasion (EMVI) (Beets-Tan et al, 2001; Brown et al, 2003). Routine use of MRI has led to the implementation of selective management strategies including surgery alone for patients with low-risk tumours ( $\leqslant \mathrm{T} 2$, N0, and no risk factors) or neoadjuvant therapy (i.e., long-course chemo-radiotherapy or short-course radiotherapy depending on the need of tumour downsizing/ downstaging) followed by surgery for those at higher risk of tumour recurrence (i.e., $\geqslant \mathrm{T} 3$ and/or $\mathrm{N}+$ stage, and/or other risk factors) (Glimelius et al, 2013; NCCN Clinical Practice Guidelines in Oncology (NCCN Guidelines), 2017).

Magnetic resonance imaging has also played an increasingly important role in the assessment of response to neoadjuvant treatment and surgical planning (Beets-Tan and Beets, 2004; MERCURY Study Group, 2007; Smith and Brown, 2008). A number of imaging parameters have been proposed to assess the degree of tumour response including linear, bi-dimensional or volumetric changes of the primary tumour and $\mathrm{T} / \mathrm{N}$ downstaging (Kim et al, 2005; Kang et al, 2010; Yeo et al, 2010; Patel et al, 2012; Yeo et al, 2012; Xiao et al, 2015). More recently, based on the ability to distinguish between areas of residual cancer and treatment-induced fibrosis (i.e., intermediate signal intensity and low signal intensity, respectively, on T2-weighted images) and estimate the relative proportion of these within the treated tumour mass, an imaging-based, tumour regression scoring system (i.e., mrTRG) has been developed (Patel et al, 2011) that follows the principles of the pathological tumour regression grading (pTRG) proposed by Mandard et al (1994) and Dworak et al (1997).

The main advantage of mrTRG is that it does not rely on the assessment of the resection specimens. The degree of tumour regression on MRI can be assessed before any surgical procedure is performed, this information potentially providing an opportunity window to consider salvage pre-operative treatments if satisfactory tumour regression is not achieved with standard therapy or adoption of a non-operative management strategy if complete response with no active residual cancer is diagnosed (Habr-Gama et al, 2004; Maas et al, 2011; Renehan et al, 2016; Sclafani and Brown, 2016; Sclafani and Chau, 2016). Furthermore, if appropriate training is undertaken, assessing tumour regression changes on imaging scans may prove to be highly reproducible (Siddiqui et al, 2016a) and potentially overcome some of the limitations of pTRG including the need for accurate pathological sampling, subjective interpretation and inter-observer variability (Lindebjerg et al, 2011; Chetty et al, 2012a, b). Nevertheless, while the prognostic value of pTRG in rectal cancer has been largely confirmed and validated across a number of patient series and using a variety of classification systems, fewer studies have analysed the association between mrTRG and outcome in this setting (Trakarnsanga et al, 2014; Siddiqui et al, 2016b). Also, little information is currently available regarding the correspondence between imaging and pathological tumour regression changes in individual patients as well as the prognostic significance of mrTRG in patients who undergo surgery and are therefore assessable for pTRG.

In a recent report of Pooled Analysis of Individual Clinical and Molecular Data of Two Trials of Neoadjuvant Chemotherapy in High-Risk Rectal Cancer, EXPERT and EXPERT-C (PAN-EX), we have shown that, in a population of high-risk, locally advanced rectal cancer patients who were treated with an intensified neoadjuvant treatment including induction chemotherapy followed by chemo-radiotherapy, both mrTRG and pTRG were prognostic for relapse-free survival (RFS), distant relapse-free survival (DRFS) and overall survival (OS) in univariate analyses (Sclafani et al,
2016). However, in multivariate analyses, only mrTRG retained its prognostic role. Here we report the correlation between mrTRG and PTRG as assessed in individual patients from this study and investigate the prognostic value of mrTRG in pTRG-defined patient subgroups.

\section{MATERIALS AND METHODS}

PAN-EX was a pooled analysis of two academic phase II trials, EXPERT and EXPERT-C (Sclafani et al, 2016). EXPERT was a single-centre, single-arm, phase II trial conducted at The Royal Marsden NHS Foundation Trust between 2001 and 2005. EXPERT-C was an international, multicentre, randomised phase II trial conducted at 12 sites across the UK, Sweden and Spain between 2005 and 2008. Both studies were restricted to nonmetastatic rectal cancer patients with at least one of the following high-risk features on high-resolution pelvic MRI: tumour $\leqslant 1 \mathrm{~mm}$ of the mesorectal fascia, extramural invasion $>5 \mathrm{~mm}(\mathrm{~T} 3 \mathrm{c} / \mathrm{d}), \mathrm{T} 4$ stage, T3 tumour at/below levators. Additional eligibility criteria included N2 stage (EXPERT) and EMVI (EXPERT-C) (Chau et al, 2006; Chua et al, 2010; Dewdney et al, 2012).

All patients received neoadjuvant treatment including four cycles of induction CAPOX chemotherapy followed by capecitabine-based chemo-radiotherapy (54 Gy in EXPERT and 50.4 Gy in EXPERT-C). Total mesorectal excision was performed 4-6 weeks after completion of chemo-radiotherapy and followed by four cycles of adjuvant chemotherapy (single agent capecitabine in EXPERT and CAPOX in EXPERT-C). Cetuximab was also administered throughout the study treatment in patients who were randomised (1:1 ratio) to the investigational arm of the EXPERT-C trial.

High-resolution MRI of the pelvis was done at baseline, at the end of induction chemotherapy and 4 weeks after completion of chemo-radiotherapy. All study MRI scans were centrally assessed by one radiologist (G.B.) who was blinded to the pathological data and long-term patient outcomes. MRI scans from EXPERT-C were prospectively assessed while MRI scans from EXPERT were retrospectively reviewed. mrTRG was defined as previously reported (Patel et al, 2011). For the assessment of pTRG, the recommendations by Dworak were followed (Dworak et al, 1997). Generally, a minimum of five blocks or 5-8 $\mathrm{mm}$ thick slices were obtained from areas of macroscopic residual tumour and suspect area of fibrotic tissue (if no macroscopic residual tumour), respectively, in formalin fixed resected specimens. Three- to 5 - $\mu \mathrm{m}$ thick, haematoxylin and eosin slides from each block were prepared and examined. If no tumour was found on the first slide a step section technique was used and three levels were cut on all blocks from the tumour site; if still no tumour was found, the whole of the tumour site and/or the fibrotic area was blocked. pTRG was assessed by local independent pathologists who were blinded to the imaging data. Assessment of pTRG was done prospectively in EXPERT-C and retrospectively in EXPERT. Both the imaging and the pathological system used to score TRG in this study assess the relative proportion of fibrosis and residual cancer and categorise tumour response using a five-tier scale in the reverse order (Table 1). Only patients who were assessable for both mrTRG after chemo-radiotherapy and pTRG on resection specimens having undergone resection of the primary tumour with a curative intent (R0/R1 resection) were included in this analysis.

EXPERT and EXPERT-C were approved by the relevant National Regulatory Agencies and Research Ethics Committee. All patients provided written informed consent. The PAN-EX study was approved by the Committee for Clinical Research at The Royal Marsden NHS Foundation Trust. 
Table 1. Classification system for mrTRG and pTRG

\begin{tabular}{|c|c|c|c|}
\hline \multicolumn{2}{|l|}{ mrTRG } & \multicolumn{2}{|r|}{ pTRG } \\
\hline Description & Grade & Grade & Description \\
\hline $\begin{array}{l}\text { No regression (intermediate signal intensity, same appearances as } \\
\text { original tumour) }\end{array}$ & mrTRG 5 & pTRG 0 & No regression \\
\hline $\begin{array}{l}\text { Slight regression (little areas of low signal intensity fibrosis or } \\
\text { mucin but mostly tumour) }\end{array}$ & mrTRG 4 & pTRG 1 & Dominant tumour mass with obvious fibrosis and/or vasculopathy \\
\hline $\begin{array}{l}\text { Moderate regression (low signal intensity fibrosis predominates } \\
\text { but there are obvious areas of intermediate signal intensity) }\end{array}$ & mrTRG 3 & pTRG 2 & $\begin{array}{l}\text { Dominantly fibrotic changes with few tumour cells or groups (easy } \\
\text { to find) }\end{array}$ \\
\hline $\begin{array}{l}\text { Good regression (predominant low signal intensity fibrosis with no } \\
\text { obvious residual tumour signal) }\end{array}$ & mrTRG 2 & pTRG 3 & $\begin{array}{l}\text { Very few (difficult to find microscopically) tumour cells in fibrotic } \\
\text { tissue with or without mucous substance }\end{array}$ \\
\hline $\begin{array}{l}\text { Complete regression (absence of tumour signal and barely visible } \\
\text { treatment related scar) }\end{array}$ & mrTRG 1 & pTRG 4 & No tumour cells, only fibrotic mass (total regression or response) \\
\hline
\end{tabular}

Statistical analysis. The strength of agreement between mrTRG after completion of neoadjuvant treatment and pTRG on the resection specimens was assessed using the weighted $\kappa$ test $(\kappa$ value $<0.20$ : poor agreement; $\kappa$ value $=0.21-0.40$ : fair agreement; $\kappa$ value $=0.41-0.60:$ moderate agreement; $\kappa$ value $=0.61-0.80:$ good agreement; $\kappa$ value $=0.81-1.00$ : very good agreement). This analysis was conducted considering the pre-defined five categories of tumour regression as well as converting the above described five-tier regression systems into three-tier regression systems (i.e., pTRG 0-1 vs pTRG 2 vs pTRG 3-4 and mrTRG 4-5 vs mrTRG 3 vs mrTRG 1-2). Furthermore, correlation between prognostic categories as defined by each regression system was also investigated in an exploratory analysis. Sensitivity, specificity, positive predictive value and negative predictive value of mrTRG were calculated using a dichotomous classification for both mrTRG (i.e., mrTRG 3-5 vs mrTRG 1-2) and pTRG (i.e., pTRG 0-3 vs pTRG 4) to assess the ability of MRI to identify pathological complete responders. Finally, the agreement between two radiologists in the assessment of mrTRG (i.e., mrTRG 3-5 vs mTRG 12) was analysed in a subset of randomly selected patients from the EXPERT-C study using the weighted $\kappa$ test as above described.

Relapse-free survival and OS were calculated from date of surgery to date of recurrence and death, respectively. Patients alive and without evidence of tumour recurrence were censored at last followup. Patients who died without tumour recurrence were censored at the time of death. The Kaplan-Meier method was used to estimate the survival outcome. Software used was SPSS 24 and Stata 14.

\section{RESULTS}

The PAN-EX study included 269 patients (105 from the EXPERT trial and 164 from the EXPERT-C trial). Of these, 240 underwent resection of the primary tumour with a curative intent (i.e., R0 resection in 233 cases while $\mathrm{R} 1$ resection in seven cases). Radiological tumour regression on post-chemo-radiotherapy MRI scan (i.e., mrTRG) and pathological tumour regression on surgical specimens (i.e., pTRG) was reported for 215 and 208 patients, respectively. A total of 191 patients (79.6\%) were assessable for both mrTRG and pTRG and represented the study population. Patient demographics and baseline characteristics are shown in Table 2. No significant differences were observed compared with the overall PAN-EX population (data not shown). The median time from the end of chemo-radiotherapy to the pre-operative MRI scan was 4.1 weeks (IQR: 3.7-4.7). The median time from the end of chemo-radiotherapy to surgery was 6.6 weeks (IQR: 5.9-7.6). The median time from the pre-operative MRI scan to surgery was 2.6 weeks (IQR: $1.7-3.7$ ).

\section{Table 2. Demographics and baseline characteristics}

\begin{tabular}{|c|c|c|}
\hline & $N$ & $\%$ \\
\hline \multicolumn{3}{|l|}{ Gender } \\
\hline Male & 108 & 56.5 \\
\hline Female & 83 & 43.5 \\
\hline Age (yrs) (median \& range) & 63 & $31-80$ \\
\hline \multicolumn{3}{|l|}{ ECOG performance status } \\
\hline 0 & 81 & 42.4 \\
\hline $1-2$ & 110 & 57.6 \\
\hline \multicolumn{3}{|l|}{ Tumour differentiation } \\
\hline Well or moderately differentiated & 163 & 85.3 \\
\hline Poorly differentiated & 20 & 10.5 \\
\hline Missing & 8 & 4.2 \\
\hline \multicolumn{3}{|l|}{ Tumour location ${ }^{a}$} \\
\hline High rectum & 69 & 36.1 \\
\hline Mid rectum & 78 & 40.8 \\
\hline Low rectum & 44 & 23.0 \\
\hline Length of tumour ${ }^{\mathrm{a}}(\mathrm{mm})$ (mean \& s.d.) & 54.4 & 15.8 \\
\hline \multicolumn{3}{|l|}{ T stage ${ }^{a}$} \\
\hline 2 & 5 & 2.6 \\
\hline $3^{b}$ & 144 & 75.4 \\
\hline 4 & 42 & 22.0 \\
\hline \multicolumn{3}{|l|}{ N stage a $^{a}$} \\
\hline 0 & 61 & 31.9 \\
\hline 1 & 64 & 33.5 \\
\hline 2 & 66 & 34.6 \\
\hline MRF threatened/involved ${ }^{a}$ & 111 & 58.1 \\
\hline EMVI positive ${ }^{a}$ & 136 & 71.2 \\
\hline \multicolumn{3}{|c|}{$\begin{array}{l}\text { Abbreviations: } E C O G=\text { Eastern Cooperative Oncology Group; } E M V I=\text { extramural venous } \\
\text { invasion; MRF = mesorectal fascia; mrTRG = magnetic resonance tumour regression grade; } \\
\text { pTRG = pathological tumour regression grade; s.d. }=\text { standard deviation. } \\
\text { a Assessed by MRI (high: }>10 \mathrm{~cm} \text { from the anal verge; mid: }>5-10 \mathrm{~cm} \text { from the anal verge; } \\
\text { low: } \leq 5 \mathrm{~cm} \text { from the anal verge). } \\
\text { b }_{117 / 144}(81.3 \% \text { ) patients with } \mathrm{T} 3 \text { tumours had }>5 \mathrm{~mm} \text { mesorectal infiltration (i.e., T3c or } \\
\text { T3d tumours). }\end{array}$} \\
\hline
\end{tabular}

In 64 out of 191 cases (33.5\%) radiologist and pathologist quantitated tumour regression using specular regression categories. Overall agreement between mrTRG and pTRG using weighted $k$ analysis was $71.0 \%(k: 0.24)$ (Table 3$)$. In the group of patients for whom disagreement was due to a substantially more pronounced regression on imaging than pathology specimens (i.e., mrTRG 1/pTRG 0-2, mrTRG 2/pTRG 0-1, mrTRG 3/pTRG $0 ; n=24)$ the median time from pre-operative MRI scan to surgery was 2.7 weeks (IQR: 2.0-3.5). In the group of patients for whom disagreement was due to a substantially more pronounced 
regression on pathology specimens than imaging (i.e., mrTRG 5/pTRG 2-4, mrTRG 4/pTRG 3-4, mrTRG 3/pTRG 4; $n=16$ ) the median time from pre-operative MRI scan to surgery was 2.1 weeks (IQR: $1.1-3.9)$. In the remaining patients $(n=151)$ this interval was 2.4 weeks (IQR: 1.7-3.7). When only three categories were considered for each regression system, 88/191 patients (46.1\%) were classified as having specular degree of tumour regression on imaging and pathology for an overall agreement of $66.0 \%(k: 0.25)$.

Previous analyses of the PAN-EX study showed that patients who had achieved mrTRG 1-2 after completion of chemoradiotherapy had a significantly better prognosis than the remaining patient population without any difference between mrTRG 3 and mrTRG 4-5. In contrast, the assessment of pathological regression with the Dworak system allowed identification of a group (pTRG 2) with an intermediate prognosis between patients with pTRG 0-1 and those with pTRG 3-4 (Sclafani et al, 2016). 44 out of 87 patients (50.6\%) belonging to the favourable prognosis group based on the degree of radiological tumour regression (mrTRG 1-2) were also found to have a good pathological tumour regression (pTRG 3-4), the remaining patients being classified as having a pTRG of $2(31.0 \%)$ or $0-1$ (18.4\%). Among 104 patients with predicted poor prognosis due to suboptimal tumour regression on pre-operative MRI scan (mrTRG 3-5), $49(47.1 \%)$ were confirmed to have minimal pathological tumour regression (pTRG 0-1), while $31(29.8 \%)$ and $24(23.1 \%)$ showed a pTRG of 2 and 3-4, respectively. When a dichotomous classification (i.e., pTRG $0-3$ vs pTRG 4 and mrTRG 3-5 vs mrTRG 1-2) was used to assess the ability of MRI to predict complete regression, 32 out of 43 patients (74.4\%) with

\section{Table 3. Comparison between mrTRG and pTRG}

\begin{tabular}{|c|c|c|c|c|c|c|c|}
\hline & \multicolumn{5}{|c|}{$\mathrm{mrTRG}^{\mathrm{a}}$} & \\
\hline & & 5 & 4 & 3 & 2 & 1 & Total \\
\hline \multirow[t]{6}{*}{$\mathrm{pTRG}^{\mathrm{b}}$} & 0 & 0 & 4 & 4 & 1 & 0 & 9 \\
\hline & 1 & 4 & 17 & 20 & 13 & 2 & 56 \\
\hline & 2 & 0 & 12 & 19 & 23 & 4 & 58 \\
\hline & 3 & 0 & 6 & 8 & 24 & 4 & 42 \\
\hline & 4 & 0 & 5 & 5 & 12 & 4 & 26 \\
\hline & Total & 4 & 44 & 56 & 73 & 14 & 191 \\
\hline
\end{tabular}

Abbreviations: $\mathrm{mrTRG}=$ magnetic resonance tumour regression grade; $\mathrm{pTRG}=$ pathological tumour regression grade.

$a_{m r T R G} 1$ and mrTRG 5 indicate complete radiological regression and no radiological regression, respectively.

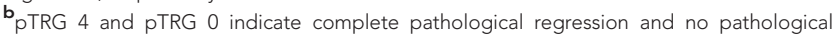
regression, respectively. pathological complete response were correctly identified using mrTRG. Sensitivity, specificity, positive predictive value and negative predictive value of $\mathrm{mrTRG}$ were $74.4 \%$ (95\% CI: 58.8 86.5), 62.8\% (95\% CI: 54.5-70.6), 36.8\% (95\% CI: 26.7-47.8) and 89.4\% (95\% CI: 81.9-94.6), respectively. MRI scans of 25 randomly selected patients from the EXPERT-C study were reviewed by two radiologists (G.B. and S.B.) to assess inter-observer variability in the assessment of complete/almost complete responders (i.e., mrTRG 1-2 vs mrTRG 3-5) and a very good agreement was observed (weighted $k$ agreement: $92.0 \%$; $k: 0.84$ ).

The median follow-up of the study population at the time when the analysis was conducted was 65.5 months (95\% CI: 65.1-66.2; range 1.5-122.0 months). Table 4 shows the outcome of pTRGdefined patient subgroups according to the degree of radiological tumour regression achieved after chemo-radiotherapy. While mrTRG did not have any influence on the good prognosis of patients with a pTRG of 3-4, it appeared to have a non-statistically significant impact especially on the outcome of those who were classified as having moderate pathological regression. In this group 5 -year RFS and OS rates were $65.9 \%$ and $68.8 \%$, respectively, if mrTRG after chemo-radiotherapy was 3-5, and 76.9\% (pairwise comparison $P$ value $=0.180$ ) and $80.6 \%$ (pairwise comparison $P$ value $=0.220)$ if $\mathrm{mrTRG}$ after chemo-radiotherapy was $1-2$. Table 5 and Figure 1 show long-term outcomes of patients who were classified by combining mrTRG and pTRG and splitting the intermediate pathological tumour regression group (pTRG 2) into two subgroups depending on the degree of tumour regression on imaging.

\section{DISCUSSION}

To our knowledge, this is the largest study assessing the agreement between an MRI-based tumour regression grading system and the classical pTRG system as proposed by Dworak et al in a largely homogenous series of locally advanced rectal cancer patients. The results of our analysis overall suggest that limited correspondence exists between regression groups/prognostic categories as identified by such classification methods. Nevertheless, they support the contention that mrTRG may potentially be used as an imaging parameter to select good-prognosis patients who could be managed with a non-operative approach following neoadjuvant treatment. Furthermore, information on the degree of tumour regression on the pre-operative MRI may be of prognostic value after surgical resection in specific pTRG patient subgroups.

Monitoring treatment efficacy has a central role in the management of cancer patients. Assessing the effects of treatment is not only important to define treatment duration but it has also the potential to

Table 4. Prognostic impact of mrTRG in specific pTRG patient subgroups

\begin{tabular}{|c|c|c|c|c|c|c|}
\hline Outcome measure & pTRG/mrTRG subgroup & $\begin{array}{c}n \text { Events/n } \\
\text { subjects }\end{array}$ & $\begin{array}{c}\text { 5-year survival } \\
(95 \% \mathrm{Cl})\end{array}$ & $\begin{array}{c}\text { Hazard ratio } \\
(95 \% \mathrm{Cl})\end{array}$ & $P$ value & Pairwise $p$ value \\
\hline \multirow{6}{*}{ RFS } & 1. pTRG $0-1 / \mathrm{mrTRG} 3-5$ & $23 / 49$ & $57.1(43.2-71.0)$ & 1.0 & $(0.023)$ & 1 vs 2 \\
\hline & 2. pTRG $0-1 / \mathrm{mr}$ TRG $1-2$ & $6 / 16$ & $73.3(50.9-95.6)$ & $0.64(0.26-1.60)$ & 0.34 & 0.391 \\
\hline & 3. pTRG $2 / m r T R G ~ 3-5$ & $11 / 31$ & $65.9(48.7-83.1)$ & $0.85(0.41-1.76)$ & 0.65 & 3 vs 4 \\
\hline & 4. pTRG $2 / m r T R G ~ 1-2$ & $6 / 27$ & $76.9(60.6-93.2)$ & $0.45(0.18-1.11)$ & 0.081 & 0.180 \\
\hline & 5. pTRG 3-4/mrTRG 3-5 & $3 / 24$ & $87.3(73.8-100)$ & $0.25(0.07-0.84)$ & 0.025 & 5 vs 6 \\
\hline & 6. pTRG 3-4/mrTRG 1-2 & $8 / 44$ & 88.5 (78.9-98.1) & $0.30(0.14-0.68)$ & 0.004 & 0.974 \\
\hline \multirow{6}{*}{ OS } & 1. pTRG $0-1 / \mathrm{mrTRG} 3-5$ & $21 / 49$ & $63.1(49.6-76.6)$ & 1.0 & $(0.053)$ & 1 vs 2 \\
\hline & 2. pTRG $0-1 / \mathrm{mr}$ TRG $1-2$ & $5 / 16$ & $80.0(59.8-100)$ & $0.57(0.21-1.52)$ & 0.260 & 0.301 \\
\hline & 3. pTRG $2 / m r T R G ~ 3-5$ & $10 / 31$ & $68.8(51.7-85.9)$ & $0.83(0.39-1.80)$ & 0.638 & 3 vs 4 \\
\hline & 4. pTRG $2 / m r T R G ~ 1-2$ & $5 / 27$ & $80.6(65.3-95.9)$ & $0.45(0.17-1.20)$ & 0.110 & 0.220 \\
\hline & 5. pTRG 3-4/mrTRG 3-5 & $3 / 24$ & $91.7(78.2-100)$ & $0.30(0.09-1.00)$ & 0.050 & 5 vs 6 \\
\hline & 6. PTRG $3-4 / m r T R G ~ 1-2$ & $7 / 44$ & $90.7(82.1-99.3)$ & $0.31(0.013-0.72)$ & 0.007 & 0.771 \\
\hline
\end{tabular}

Abbreviations: $\mathrm{Cl}=$ confidence interval; $\mathrm{mrTRG}=$ magnetic resonance tumour regression grade; $\mathrm{OS}=$ overall survival; $\mathrm{pTRG}=$ pathological tumour regression grade; $\mathrm{RFS}=$ recurrence-free survival. 
Table 5. Survival outcomes by combining mrTRG and PTRG

\begin{tabular}{|c|c|c|c|c|c|}
\hline Outcome measure & pTRG/mrTRG subgroup & $n$ Events/n subjects & 5-year survival $(95 \% \mathrm{Cl})$ & Hazard ratio $(95 \% \mathrm{Cl})$ & $P$ value \\
\hline RFS & $\begin{array}{l}\text { PTRG 0-1/mrTRG any } \\
\text { PTRG 2/mrTRG 3-5 } \\
\text { pTRG 2/mrTRG 1-2 } \\
\text { pTRG 3-4/mrTRG any }\end{array}$ & $\begin{array}{l}40 / 96 \\
17 / 95\end{array}$ & $\begin{array}{l}62.7(52.9-72.5) \\
86.0(78.9-93.1)\end{array}$ & $\begin{array}{c}1.0 \\
0.37(0.21-0.66)\end{array}$ & 0.001 \\
\hline os & $\begin{array}{l}\text { pTRG 0-1/mrTRG any } \\
\text { pTRG 2/mrTRG 3-5 } \\
\text { pTRG 2/mrTRG 1-2 } \\
\text { pTRG 3-4/mrTRG any }\end{array}$ & $\begin{array}{l}36 / 96 \\
15 / 95\end{array}$ & $\begin{array}{l}67.5(57.9-77.1) \\
88.1(81.4-94.8)\end{array}$ & $\begin{array}{c}1.0 \\
0.39(0.22-0.72)\end{array}$ & 0.003 \\
\hline
\end{tabular}

A

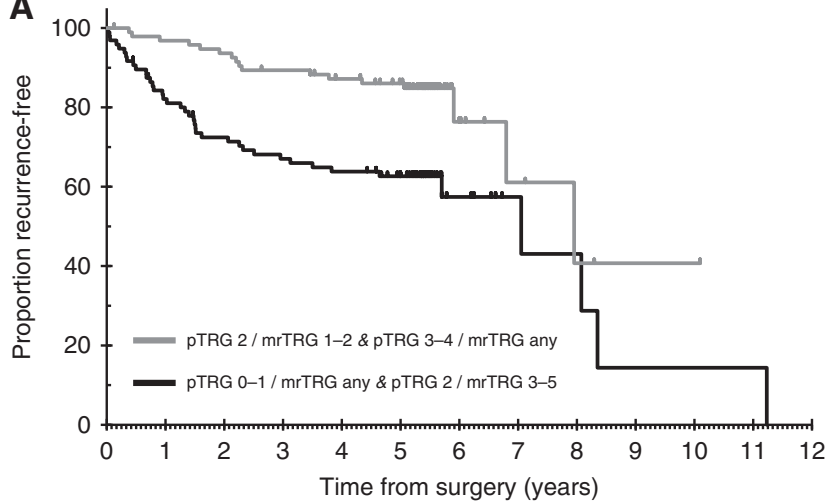

$N$ at risk:
B

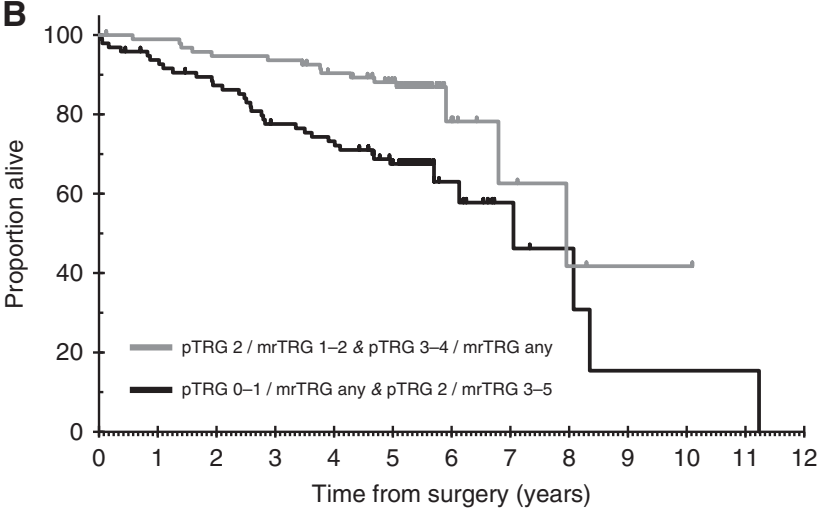

$N$ at risk:

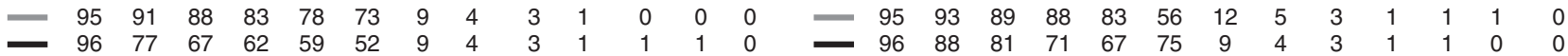

Figure 1. Kaplan-Meier curves for recurrence-free survival (A) and overall survival (B) by combining mrTRG and pTRG.

provide useful prognostic information and guide subsequent therapeutic and/or follow-up strategies. This is certainly relevant to rectal cancer where neoadjuvant therapy is routinely used for locally advanced tumours to increase the chances of surgical resection with clear margins and/or to reduce the risk of local recurrence (Braendengen et al, 2008; Sauer et al, 2012). Meticulous assessment of the surgical samples was proposed more than two decades ago to provide a semi-quantitative estimate of the degree of tumour regression with prognostic implications (Mandard et al, 1994; Dworak et al, 1997) and pTRG is now regularly included in pathology reports. Nevertheless, its clinical usefulness remains limited for a number of reasons. First of all, assessment of response to neoadjuvant treatment by pTRG is only possible in patients who undergo resection and only after a surgical procedure has been performed. Furthermore, the potential of pTRG to capture the heterogeneity of tumour response to chemo-radiotherapy and overcome the simplistic distinction between complete responders and noncomplete responders has inevitably translated into a relatively low inter-observer reproducibility of the same (Lindebjerg et al, 2011; Chetty et al, 2012a, b). The availability of a variety of pTRG scoring systems has enhanced this inconvenience and also reduced comparability between series. Finally, despite a promising premise, the impact of pTRG on the management of rectal cancer is still limited as there is still no evidence to suggest that tailoring postoperative treatments based on the degree of pathological regression may improve outcome.

Advances in imaging have provided an opportunity to use novel, alternative methods for the assessment of tumour response to neoadjuvant chemo-radiotherapy. mrTRG has recently emerged as a dynamic, non-invasive, surrogate method for the assessment of tumour regression following neoadjuvant treatment and before surgical resection (Patel et al, 2011). Studies have shown its ability to predict pathological findings on the surgical specimens as well as long-term prognosis (Patel et al, 2011; Shihab et al, 2011; Bhoday et al, 2016; Siddiqui et al, 2016b). Also, both in routine practice (Sclafani et al, 2016) and adaptive clinical trials (NCT02704520) mrTRG has been increasingly used as a decision-making tool for patient management following completion of standard neoadjuvant chemo-radiotherapy.

Nevertheless, one of the questions that remain unanswered regarding mrTRG is whether and to what extent this truly reflects the degree of pathological regression that will be observed once surgery has been carried out. In this study we have shown that, even though tumour regression on imaging is measured according to a five-tier scoring system mirroring the scoring system that is used for the assessment of pathological regression, the agreement between radiologists and pathologists is overall limited. This did not appear to be any better when regression groups with similar prognosis across the two scoring systems (instead of the predefined categories of regression) were directly compared. While these results are in line with other studies (van den Broek et al, 2017) and appear to suggest that mrTRG cannot be considered as an accurate predictor of pTRG, it should be noted that the time interval between chemo-radiotherapy and surgery in our series was longer than that between chemo-radiotherapy and MRI (difference between median values: 2.5 weeks) this possibly accounting for some of the observed discrepancies. Response to chemo-radiotherapy is a continuum process with the peak of tumour regression being estimated to occur $\sim 8-11$ weeks after treatment completion (Sloothaak et al, 2013; Probst et al, 2015; Sun et al, 2016). Therefore, 
it is possible that the early timing of MRI assessment in this patient population may have precluded the anticipated category shift in tumour regression and ultimately affected the agreement between mrTRG and pTRG. In support of this contention, the proportion of study patients achieving complete regression on resection specimens was almost twice as high as that on imaging (i.e., $13.6 \%$ vs $7.3 \%$ ). On the other hand, while cases where poor pathological regression was reported despite complete or almost complete absence of intermediate signal intensity on pre-operative imaging could raise the possibility of early tumour regrowth, the median interval between MRI scan and surgical resection in this patient subgroup (2.7 weeks vs 2.5 in the overall population) mitigates against this hypothesis. We believe that these cases are more likely to be secondary to a reduced specificity of MRI after neoadjuvant treatment (Memon et al, 2015). Finally, it is also possible that the investigational, intensified neoadjuvant treatment strategies that were used in the PAN-EX study might have had an impact on the limited correspondence between mrTRG and pTRG. For instance, in contrast with what we have previously reported in studies with conventional chemo-radiotherapy (Patel et al, 2011), only two (instead of three) mrTRG prognostic groups could be identified in the PAN-EX population where a regression category with intermediate prognosis was lacking (Sclafani et al, 2016).

Even accounting for the above mentioned biases, one could argue that the lack of substantial correspondence between tumour regression as identified by imaging and pathology may undermine the clinical value of mrTRG. It should be noted, however, that the prognostic role of this imaging parameter has already been validated in a number of series and in the PAN-EX study itself mrTRG (but not pTRG) emerged as an independent factor for RFS, DRFS and OS in the curatively resected population (Shihab et al, 2011; Bhoday et al, 2016; Sclafani et al, 2016; Siddiqui et al, 2016b). Moreover, the main value of using mrTRG is to provide a non-invasive imaging tool to identify those patients who are more likely to achieve complete response to treatment and therefore may be spared from surgery (Habr-Gama et al, 2004; Maas et al, 2011; Renehan et al, 2016). In our series, the sensitivity and specificity of mrTRG to identify pathological complete response was $74.4 \%$ and $62.8 \%$, respectively. While these data appear less encouraging compared to previous reports (Bhoday et al, 2016), it should be noted that in this study more stringent criteria (i.e., mrTRG 1-2) for the definition of complete response according to imaging were used excluding mrTRG 3 tumours that may actually continue to regress beyond the date of MRI scan. Furthermore, repeat assessment of mrTRG on serial MRI scans to follow the evolution of tumour regression over time is more clinically relevant than a snapshot of mrTRG at a single time point and likely to increase the overall accuracy of this imaging marker in predicting pathological complete response. In this regard it is worth noting that, using a subset of study patients, we have confirmed the results of previous studies regarding the reproducibility of mrTRG (Siddiqui et al, 2016a) and showed a very good agreement between radiologists in the assessment of complete or almost complete tumour regression.

The results of our study suggest that, despite similar nomenclature and categorisation system, mrTRG and pTRG are not interchangeable parameters but have the potential to provide complementary information regarding sensitivity of the primary tumour to neoadjuvant treatment and overall outcome. Of interest, patients who had not achieved substantial/complete regression according to the pathology report could be further stratified for the risk of tumour recurrence and death based on the degree of tumour regression as assessed on imaging at the end of chemo-radiotherapy. This effect appeared possibly stronger for those who were categorised as having a pTRG of 2 and therefore expected to have intermediate prognosis. While these data are certainly intriguing, it should be acknowledged that the impact of mrTRG on the prognosis of patients with sub-optimal pathological regression was only numerical but not statistically significant possibly due to the small sample size of the study. Larger series are needed to confirm these hypothesis-generating findings and better assess the clinical relevance of mrTRG in patients who undergo surgical resection and are therefore assessable for standard pTRG.

Intra- and inter-observer reproducibility is an established caveat of semi-quantitative scoring systems. A number of studies have demonstrated that achieving consensus on the degree of tumour regression in resection specimens among pathologists is particularly challenging (Lindebjerg et al, 2011; Chetty et al, 2012a, b). While the assessment of pTRG by local pathologists suggests that the pathological data from PAN-EX are reflective of real-world practice, the lack of a centralised review (in contrast with the radiological assessment) may have introduced significant biases. mrTRG appears to be less prone to inter-observer variability among specifically trained radiologists (Patel et al, 2011), as confirmed by the analysis of a subset of patients from our study. The assessment of mrTRG, however, may be still potentially affected by a certain degree of subjective interpretation. Therefore, the results of our study should be interpreted in view of these inherent limitations. It is possible that the findings here reported may not be confirmed in series from other institutions. Nevertheless, the mrTRG scoring system, which was first proposed by our group, has been routinely used for several years at our institution and pTRG in PAN-EX was assessed by highly experienced pathologists, thus increasing the reliability of our study. Also, the use of a weighted $k$ test to compare radiological and pathological assessment should have accounted for some interobserver variability and increased the generalisability of our results. Finally, given the similarities between all the available pTRG scoring systems both in terms of classification criteria and prognostication (Trakarnsanga et al, 2014), it is unlikely that classifying pathological tumour regression according to a different method would change the outcome of the analysis.

In conclusion, while the retrospective design of the study and the above mentioned caveats recommend caution in the interpretation of our findings, this analysis suggests that the agreement between mrTRG and pTRG is low and thus mrTRG cannot be considered as a reliable surrogate imaging marker of pTRG in locally advanced rectal cancer patients who undergo an intensified neoadjuvant treatment. Nevertheless, given the ability to provide a non-invasive assessment of tumour response, mrTRG remains a potential tool for the implementation of dynamic, risk-adapted, neoadjuvant treatment strategies following standard chemo-radiotherapy including deferral of surgery/watch and wait or further (i.e., sequential/salvage) therapy. Assessing the degree of tumour regression on pre-operative MRI scans may potentially serve also as a useful parameter to combine with conventional pathological factors to refine prognostication and possibly guide adjuvant treatments/follow-up strategies after surgical resection. It should be noted, however, that mrTRG is only one of the available methods to assess and categorise tumour response to neoadjuvant treatment and its significance should be interpreted in the context of other well-established imaging parameters. Furthermore, mrTRG is regularly reported only in a limited number of centres and further studies to investigate its clinical applications are warranted.

\section{ACKNOWLEDGEMENTS}

The work was supported by the National Institute for Health Research (NIHR) Biomedical Research Centre (BRC) at The Royal Marsden NHS Foundation Trust and The Institute of Cancer Research. The EXPERT study was supported by a fellowship grant from the Pelican Cancer Foundation and by an education grant from Sanofi-Aventis which also provided the study drug. The EXPERT-C trial was endorsed by Cancer Research UK and was 
supported by a research grant from Merck \& Co. Sanofi-Aventis and Merck \& Co. provided the study drugs. Neither company was involved in study design, data analysis, or manuscript preparation or had access to study data.

\section{CONFLICT OF INTEREST}

DC received research funding from: Roche, Amgen, Celgene, Sanofi, Merck Serono, Novartis, AstraZeneca, Bayer, Merrimack and MedImmune. CP has had advisory roles with Sanofi. JT has had advisory roles with Amgen, Roche, Sanofi-Aventis, and Merck. IC has had advisory roles with Merck Serono, Roche, Sanofi Oncology, Bristol Myers Squibb, Eli-Lilly, Novartis, Gilead Science. $\mathrm{He}$ has received research funding from Merck-Serono, Novartis, Roche and Sanofi Oncology, and honoraria from Roche, SanofiOncology, Eli-Lilly, Taiho. All other authors declare that they have no conflicts of interest.

\section{REFERENCES}

Magnetic Resonance Tumour Regression Grade as Biomarker for Stratified Management of Rectal Cancer Patients (TRIGGER). Available at https:// clinicaltrials.gov/ct2/show/NCT02704520.

Beets-Tan RG, Beets GL (2004) Rectal cancer: review with emphasis on MR imaging. Radiology 232(2): 335-346.

Beets-Tan RG, Beets GL, Vliegen RF, Kessels AG, Van Boven H, De Bruine A, von Meyenfeldt MF, Baeten CG, van Engelshoven JM (2001) Accuracy of magnetic resonance imaging in prediction of tumour-free resection margin in rectal cancer surgery. Lancet 357(9255): 497-504.

Bhoday J, Smith F, Siddiqui MR, Balyasnikova S, Swift RI, Perez R, Habr-Gama A, Brown G (2016) Magnetic resonance tumor regression grade and residual mucosal abnormality as predictors for pathological complete response in rectal cancer postneoadjuvant chemoradiotherapy. Dis Colon Rectum 59(10): 925-933.

Braendengen M, Tveit KM, Berglund A, Birkemeyer E, Frykholm G, Påhlman L, Wiig JN, Byström P, Bujko K, Glimelius B (2008) Randomized phase III study comparing preoperative radiotherapy with chemoradiotherapy in nonresectable rectal cancer. J Clin Oncol 26(22): 3687-3694.

Brown G, Radcliffe AG, Newcombe RG, Dallimore NS, Bourne MW, Williams GT (2003) Preoperative assessment of prognostic factors in rectal cancer using high-resolution magnetic resonance imaging. $\mathrm{Br} J \mathrm{Surg}$ 90(3): 355-364.

Chau I, Brown G, Cunningham D, Tait D, Wotherspoon A, Norman AR, Tebbutt N, Hill M, Ross PJ, Massey A, Oates J (2006) Neoadjuvant capecitabine and oxaliplatin followed by synchronous chemoradiation and total mesorectal excision in magnetic resonance imaging-defined poor-risk rectal cancer. J Clin Oncol 24(4): 668-674.

Chetty R, Gill P, Govender D, Bateman A, Chang HJ, Deshpande V, Driman D, Gomez M, Greywoode G, Jaynes E, Lee CS, Locketz M, Rowsell C, Rullier A, Serra S, Shepherd N, Szentgyorgyi E, Vajpeyi R, Wang LM, Bateman A (2012a) International study group on rectal cancer regression grading: interobserver variability with commonly used regression grading systems. Hum Pathol 43(11): 1917-1923.

Chetty R, Gill P, Govender D, Bateman A, Chang HJ, Driman D, Duthie F, Gomez M, Jaynes E, Lee CS, Locketz M, Mescoli C, Rowsell C, Rullier A, Serra S, Shepherd N, Szentgyorgyi E, Vajpeyi R, Wang LM (2012b) A multi-centre pathologist survey on pathological processing and regression grading of colorectal cancer resection specimens treated by neoadjuvant chemoradiation. Virchows Arch 460(2): 151-155.

Chua YJ, Barbachano Y, Cunningham D, Oates JR, Brown G, Wotherspoon A, Tait D, Massey A, Tebbutt NC, Chau I (2010) Neoadjuvant capecitabine and oxaliplatin before chemoradiotherapy and total mesorectal excision in MRI-defined poor-risk rectal cancer: a phase 2 trial. Lancet Oncol 11(3): 241-248.

Dewdney A, Cunningham D, Tabernero J, Capdevila J, Glimelius B, Cervantes A, Tait D, Brown G, Wotherspoon A, Gonzalez de Castro D, Chua YJ, Wong R, Barbachano Y, Oates J, Chau I (2012) Multicenter randomized phase II clinical trial comparing neoadjuvant oxaliplatin, capecitabine, and preoperative radiotherapy with or without cetuximab followed by total mesorectal excision in patients with high-risk rectal cancer (EXPERT-C). J Clin Oncol 30(14): 1620-1627.

Dworak O, Keilholz L, Hoffmann A (1997) Pathological features of rectal cancer after preoperative radiochemotherapy. Int J Colorectal Dis 12(1): 19-23.

Glimelius B, Tiret E, Cervantes A, Arnold D. ESMO Guidelines Working Group (2013) Rectal cancer: ESMO clinical practice guidelines for diagnosis, treatment and follow-up. Ann Oncol 24(Suppl 6): vi81-vi88.

Habr-Gama A, Perez RO, Nadalin W, Sabbaga J, Ribeiro Jr U, Silva e Sousa Jr AH, Campos FG, Kiss DR, Gama-Rodrigues J (2004) Operative vs nonoperative treatment for stage 0 distal rectal cancer following chemoradiation therapy: long-term results. Ann Surg 240(4): 711-717.

Kang JH, Kim YC, Kim H, Kim YW, Hur H, Kim JS, Min BS, Kim H, Lim JS, Seong J, Keum KC, Kim NK (2010) Tumor volume changes assessed by three-dimensional magnetic resonance volumetry in rectal cancer patients after preoperative chemoradiation: the impact of the volume reduction ratio on the prediction of pathologic complete response. Int J Radiat Oncol Biol Phys 76(4): 1018-1025.

Kim YH, Kim DY, Kim TH, Jung KH, Chang HJ, Jeong SY, Sohn DK, Choi HS, Ahn JB, Kim DH, Lim SB, Lee JS, Park JG (2005) Usefulness of magnetic resonance volumetric evaluation in predicting response to preoperative concurrent chemoradiotherapy in patients with resectable rectal cancer. Int J Radiat Oncol Biol Phys 62(3): 761-768.

Lindebjerg J, Hansborg N, Ploen J, Rafaelsen S, Jorgensen JC, Jakobsen A (2011) Factors influencing reproducibility of tumour regression grading after high-dose chemoradiation of locally advanced rectal cancer. Histopathology 59(1): 18-21.

Maas M, Beets-Tan RG, Lambregts DM, Lammering G, Nelemans PJ, Engelen SM, van Dam RM, Jansen RL, Sosef M, Leijtens JW, Hulsewé KW, Buijsen J, Beets GL (2011) Wait-and-see policy for clinical complete responders after chemoradiation for rectal cancer. J Clin Oncol 29(35): 4633-4640.

Mandard AM, Dalibard F, Mandard JC, Marnay J, Henry-Amar M, Petiot JF, Roussel A, Jacob JH, Segol P, Samama G, Ollivier JM, Bonvalot S, Gignoux M (1994) Pathologic assessment of tumor regression after preoperative chemoradiotherapy of esophageal carcinoma. Clinicopathologic correlations. Cancer 73(11): 2680-2686.

Memon S, Lynch AC, Bressel M, Wise AG, Heriot AG (2015) Systematic review and meta-analysis of the accuracy of MRI and endorectal ultrasound in the restaging and response assessment of rectal cancer following neoadjuvant therapy. Colorectal Dis 17(9): 748-761.

MERCURY Study Group (2007) Extramural depth of tumor invasion at thin section MR in patients with rectal cancer: results of the MERCURY study. Radiology 243(1): 132-139.

NCCN Clinical Practice Guidelines in Oncology (NCCN Guidelines) (2017) Rectal Cancer. Version 2. Available at https://www.nccn.org/professionals/ physician_gls/pdf/rectal.pdf.

Patel UB, Brown G, Rutten H, West N, Sebag-Montefiore D, Glynne-Jones R, Rullier E, Peeters M, Van Cutsem E, Ricci S, Van de Velde C, Kjell P, Quirke P (2012) Comparison of magnetic resonance imaging and histopathological response to chemoradiotherapy in locally advanced rectal cancer. Ann Surg Oncol 19(9): 2842-2852.

Patel UB, Taylor F, Blomqvist L, George C, Evans H, Tekkis P, Quirke P, Sebag-Montefiore D, Moran B, Heald R, Guthrie A, Bees N, Swift I, Pennert K, Brown G (2011) Magnetic resonance imaging-detected tumor response for locally advanced rectal cancer predicts survival outcomes: MERCURY experience. J Clin Oncol 29(28): 3753-3760.

Probst CP, Becerra AZ, Aquina CT, Tejani MA, Wexner SD, Garcia-Aguilar J, Remzi FH, Dietz DW, Monson JR, Fleming FJ. Consortium for Optimizing the Surgical Treatment of Rectal Cancer (OSTRiCh) (2015) Extended intervals after neoadjuvant therapy in locally advanced rectal cancer: the key to improved tumor response and potential organ preservation. J Am Coll Surg 221(2): 430-440.

Renehan AG, Malcomson L, Emsley R, Gollins S, Maw A, Myint AS, Rooney PS, Susnerwala S, Blower A, Saunders MP, Wilson MS, Scott N, O'Dwyer ST (2016) Watch-and-wait approach vs surgical resection after chemoradiotherapy for patients with rectal cancer (the OnCoRe project): a propensity-score matched cohort analysis. Lancet Oncol 17(2): 174-183.

Sauer R, Liersch T, Merkel S, Fietkau R, Hohenberger W, Hess C, Becker H, Raab HR, Villanueva MT, Witzigmann H, Wittekind C, Beissbarth T, Rödel C (2012) Preoperative $v s$ postoperative chemoradiotherapy for locally advanced rectal cancer: results of the German CAO/ARO/AIO-94 
randomized phase III trial after a median follow-up of 11 years. J Clin Oncol 30(16): 1926-1933.

Sclafani F, Brown G (2016) Extramural venous invasion (EMVI) and tumour regression grading (TRG) as potential prognostic factors for risk stratification and treatment decision in rectal cancer. Curr Colorectal Cancer Rep 12: 130.

Sclafani F, Brown G, Cunningham D, Wotherspoon A, Tait D, Peckitt C, Evans J, Yu S, Sena Teixeira Mendes L, Tabernero J, Glimelius B, Cervantes A, Thomas J, Begum R, Oates J, Chau I (2016) PAN-EX: a pooled analysis of two trials of neoadjuvant chemotherapy followed by chemoradiotherapy in MRI-defined, locally advanced rectal cancer. Ann Oncol 27(8): 155-1565.

Sclafani F, Chau I (2016) Timing of therapies in the multidisciplinary treatment of locally advanced rectal cancer: available evidence and implications for routine practice. Semin Radiat Oncol 26(3): 176-185.

Shihab OC, Taylor F, Salerno G, Heald RJ, Quirke P, Moran BJ, Brown G (2011) MRI predictive factors for long-term outcomes of low rectal tumours. Ann Surg Oncol 18(12): 3278-3284.

Siddiqui MR, Bhoday J, Battersby NJ, Chand M, West NP, Abulafi AM, Tekkis PP, Brown G (2016b) Defining response to radiotherapy in rectal cancer using magnetic resonance imaging and histopathological scales. World J Gastroenterol 22(37): 8414-8434.

Siddiqui MR, Gormly KL, Bhoday J, Balyansikova S, Battersby NJ, Chand M, Rao S, Tekkis P, Abulafi AM, Brown G (2016a) Interobserver agreement of radiologists assessing the response of rectal cancers to preoperative chemoradiation using the MRI tumour regression grading (mrTRG). Clin Radiol 71(9): 854-862.

Sloothaak DA, Geijsen DE, van Leersum NJ, Punt CJ, Buskens CJ, Bemelman WA, Tanis PJ. Dutch Surgical Colorectal Audit (2013) Optimal time interval between neoadjuvant chemoradiotherapy and surgery for rectal cancer. Br J Surg 100(7): 933-939.

Smith N, Brown G (2008) Preoperative staging of rectal cancer. Acta Oncol 47(1): 20-31.
Sun Z, Adam MA, Kim J, Shenoi M, Migaly J, Mantyh CR (2016) Optimal timing to surgery after neoadjuvant chemoradiotherapy for locally advanced rectal cancer. Am J Coll Surg 222(4): 367-374.

Trakarnsanga A, Gönen M, Shia J, Nash GM, Temple LK, Guillem JG, Paty PB, Goodman KA, Wu A, Gollub M, Segal N, Saltz L, Garcia-Aguilar J, Weiser MR (2014) Comparison of tumor regression grade systems for locally advanced rectal cancer after multimodality treatment. J Natl Cancer Inst 106(10): pii: dju248.

van den Broek JJ, van der Wolf FS, Lahaye MJ, Heijnen LA, Meischl C, Heitbrink MA, Schreurs WH (2017) Accuracy of MRI in restaging locally advanced rectal cancer after preoperative chemoradiation. Dis Colon Rectum 60(3): 274-283.

Xiao J, Tan Y, Li W, Gong J, Zhou Z, Huang Y, Zheng J, Deng Y, Wang L, Peng J, Ren D, Lan P, Wang J (2015) Tumor volume reduction rate is superior to RECIST for predicting the pathological response of rectal cancer treated with neoadjuvant chemoradiation: results from a prospective study. Oncol Lett 9(6): 2680-2686.

Yeo SG, Kim DY, Kim TH, Jung KH, Hong YS, Chang HJ, Park JW, Lim SB, Choi HS, Jeong SY (2010) Tumor volume reduction rate measured by magnetic resonance volumetry correlated with pathologic tumor response of preoperative chemoradiotherapy for rectal cancer. Int J Radiat Oncol Biol Phys 78(1): 164-171.

Yeo SG, Kim DY, Park JW, Oh JH, Kim SY, Chang HJ, Kim TH, Kim BC, Sohn DK, Kim MJ (2012) Tumor volume reduction rate after preoperative chemoradiotherapy as a prognostic factor in locally advanced rectal cancer. Int J Radiat Oncol Biol Phys 82(2): e193-e199.

This work is published under the standard license to publish agreement. After 12 months the work will become freely available and the license terms will switch to a Creative Commons AttributionNonCommercial-Share Alike 4.0 Unported License. 